\title{
Improving health care quality and safety: the role of collective learning
}

\author{
This article was published in the following Dove Press journal: \\ Journal of Healthcare Leadership \\ 6 November 2015 \\ Number of times this article has been viewed
}

\section{Sara J Singer ${ }^{1-4}$ \\ Justin K Benzer ${ }^{4-6}$ \\ Sami U Hamdan ${ }^{4,6}$}

'Department of Health Policy and Management, Harvard T.H. Chan School of Public Health, Boston, MA, USA; ${ }^{2}$ Department of Medicine, Harvard Medical School, Boston, MA, USA; ${ }^{3}$ Mongan Institute for Health Policy, Massachusetts General Hospital, Boston, MA, USA; ${ }^{4}$ Center for Healthcare Organization and Implementation Research, VA Boston Healthcare System, Boston, MA, USA; ${ }^{5}$ VISN 17 Center of Excellence for Research on Returning War Veterans, Waco, TX, USA; ${ }^{6}$ Department of Health Policy and Management, Boston University School of Public Health, Boston, MA, USA
Correspondence: Sara J Singer, Department of Health Policy and Management, Harvard T.H. Chan School of Public Health, 677 Huntington Avenue, Boston, MA 02115, USA

Email ssinger@hsph.harvard.edu
Abstract: Despite decades of effort to improve quality and safety in health care, this goal feels increasingly elusive. Successful examples of improvement are infrequently replicated. This scoping review synthesizes 76 empirical or conceptual studies (out of 1208 originally screened) addressing learning in quality or safety improvement, that were published in selected health care and management journals between January 2000 and December 2014 to deepen understanding of the role that collective learning plays in quality and safety improvement. We categorize learning activities using a theoretical model that shows how leadership and environmental factors support collective learning processes and practices, and in turn team and organizational improvement outcomes. By focusing on quality and safety improvement, our review elaborates the premise of learning theory that leadership, environment, and processes combine to create conditions that promote learning. Specifically, we found that learning for quality and safety improvement includes experimentation (including deliberate experimentation, improvisation, learning from failures, exploration, and exploitation), internal and external knowledge acquisition, performance monitoring and comparison, and training. Supportive learning environments are characterized by team characteristics like psychological safety, appreciation of differences, openness to new ideas social motivation, and team autonomy; team contextual factors including learning resources like time for reflection, access to knowledge, organizational capabilities; incentives; and organizational culture, strategy, and structure; and external environmental factors including institutional pressures, environmental dynamism and competitiveness and learning collaboratives. Lastly learning in the context of quality and safety improvement requires leadership that reinforces learning through actions and behaviors that affect people, such as coaching and trust building, and through influencing contextual factors, including providing resources, developing culture, and taking strategic actions that support improvement. Our review highlights the importance of leadership in both promoting a supportive learning environment and implementing learning processes.

Keywords: collective learning, systematic review, scoping review, health care quality, patient safety, quality improvement

\section{Introduction}

Delivering high quality, highly reliable health care requires continuous improvement. ${ }^{1}$ Despite decades of quality and safety improvement initiatives, frequent reports of medical errors, ${ }^{2}$ failures to implement evidence-based practice, ${ }^{3}$ unnecessary variations ${ }^{4,5}$ and disparities in care, ${ }^{6}$ and redundant and wasted resources, ${ }^{7,8}$ often make achieving this goal feel increasingly elusive.

The literature is replete with examples of successful quality and safety improvement initiatives. ${ }^{9,10}$ Yet, most examples tend to be local and are infrequently replicated 
by other organizations. ${ }^{11,12}$ One reason for problems with transferability may be the lack of understanding about the mechanisms through which organizations achieve improvement. ${ }^{13,14}$ In part this stems from the absence of studies that integrate across multiple improvement initiatives. For example, research suggests that collective learning plays a role in improvement. ${ }^{15}$ By collective learning, we mean a process of gaining information, understanding, or capabilities in groups and organizations. ${ }^{16}$ Collective learning differs from individual learning because it requires individuals to interactively analyze and interpret organizational experience. ${ }^{17}$ As this review will demonstrate, individual studies of quality and safety improvement initiatives discuss the role that collective learning plays in achieving desired outcomes. Yet, no research has systematically investigated the link between collective learning and quality and safety improvement. A greater understanding of how learning promotes quality and safety may make lessons from quality improvement (QI) initiatives more actionable.

The goal of this review is to provide health care leaders and researchers with an understanding of the role that collective learning plays in quality and safety improvement. We categorize learning activities using a theoretical model that shows how leadership and environmental factors support learning processes and practices to promote collective learning and in turn team and organizational improvement outcomes. We focus specifically on learning in recognition of the importance of learning as a basic mechanism through which quality and safety are improved. A deeper understanding of the ways in which learning fosters improvement provides a more actionable foundation for continuous improvement.

\section{Building blocks of organizational learning for quality and safety improvement}

We focus on collective learning as the foundation upon which health care organizations can accomplish quality and safety improvement. To date, however, the ways in which learning facilitates improvement have been imprecisely specified. Our review draws on an existing, empirically-grounded, theoretical model of organizational learning to suggest that quality and safety improvement benefits from the presence of three building blocks of organizational learning - learning processes and practices, a supportive learning environment, and leadership that reinforces learning. As the metaphor implies, the leadership and environmental blocks build on each other to support learning processes and practices. The combination of blocks creates optimal conditions for learning that promotes improvement. However, each block independently contributes to the ability of groups to learn to improve. Through various forums and information systems, learning processes and practices provide the ability for health care organizations to learn and improve; policies and structures help create the environment that enables learning; and through actions and reactions, leaders at all levels of an organization maintain motivation among the health care workforce for continuous learning and improvement. In applying this model to a wide range of quality and safety improvement initiatives, we substantially extend the model and ground it in empirical examples.

In this review, we blend disparate research on organizational learning in quality and safety improvement efforts to highlight specific ways in which learning contributes to these efforts. Our primary contribution is the conceptual categorization of learning mechanisms and the identification of relationships among them. This is important because the fragmented nature of individual studies on organizational learning and quality and safety improvement provides an inadequate foundation for health care leaders and practitioners to pursue comprehensive learning-oriented solutions. Our analysis provides researchers with a richer, empirically and theoretically grounded framework for understanding how collective learning promotes quality and safety improvement and how learning can occur. We also offer health care leaders a guide to more effectively promoting quality and safety improvement. Our review supports the original premise of learning theory, that leadership, environment, and processes combined create conditions that promote learning, and extends this theory to quality and safety improvement.

\section{Methods}

\section{Literature review}

This paper takes the form of a scoping review in that it identifies the organizational learning concepts and activities that have been studied in the context of patient safety and QI. The purpose of a scoping review is to rapidly map the key concepts in a research area. Scoping reviews are particularly important in complex areas that have not previously been mapped. ${ }^{18,19}$ A scoping review is appropriate for organizational learning in patient safety and QI because the findings are likely to be spread across multiple disciplines in both the management and health care literature, and because the key concepts have not previously been mapped.

\section{Sources and search terms}

We used PubMed and Business Source Complete between January 2000 and December 2014 to identify relevant studies. Keywords were synonyms of organizational learning 
(ie, organization learning, organizational learning, group learning, team learning). For health care we also included the MESH term organizational innovation. For the management literature we included the keyword knowledge management. We then selected the articles that also had one of the following keywords: process improvement, QI, safety improvement, innovation, or intervention. We limited our search to top tier journals and journals that specialized in patient safety and QI. The assumption underlying this strategy is that the key organizational learning concepts would be discussed in these journals. The health care journals were: BMJ, BMJ Quality and Safety, Health Affairs, Health Care Management Review, Health Services Research, Implementation Science, JAMA, Medical Care, Medical Care Research and Review, Milbank, and NEJM. The management journals were: Academy of Management Journal, Administrative Science Quarterly, Management Science, and Organization Science.

\section{Article screening and criteria}

Our search strategy generated 1,208 abstracts, 558 in health care journals and 650 in management journals. Articles with clearly irrelevant titles were excluded. Three authors reviewed 227 full text articles and determined which articles should be included for review. Criteria for inclusion were: 1) the article must discuss learning, 2) the article must discuss either quality or safety improvement, 3) the article must be an empirical study, and 4) due to the low number of empirical studies of organizational learning in the health care literature, we included conceptual health care articles. We retained a total of 76 articles for analysis.

\section{Analysis}

Following the conceptual framework, we collectively established whether articles addressed at least one building block: learning processes, the supportive learning environment, or leadership that reinforces learning. We then assigned articles to subcategories, drawing to the extent possible on established concepts. We then divided the three building blocks; each author took primary responsibility for conducting a second review of the articles assigned to the given block, coding the articles into more detailed categories, and elaborating the learning concepts. The group discussed papers that raised questions at each stage and jointly resolved their classification, enabling integration of findings across blocks and subcategories. Table 1 summarizes the literature in each block and category. Below, we describe in detail each of the learning activities that comprise the building blocks of organizational learning that promote quality and safety improvement.

\section{Learning processes and practices}

Learning requires concrete processes and practices. In reviewing studies linking collective learning with quality and safety improvement, four types of learning processes and practices emerged. These include experimentation to develop and test new ways of doing things; acquisition of knowledge from experts, customers, and the organization's own experience; monitoring and comparing performance data; and training to develop workers' skills.

\section{Experimentation}

Experimentation refers to the extent to which a unit develops and tests new ways of doing things. ${ }^{20}$ Several studies in health care and non-health settings highlight the importance of experimentation..$^{21-32}$ Experimentation is needed to slow health spending growth and unlock innovation in health delivery. ${ }^{33}$ Experimentation with new ways of doing things in workgroups can require taking risks. ${ }^{25}$ Because human lives are at stake, health care professionals may be inclined to experiment less than in lower risk settings. Finding safe ways to experiment, such as through simulated trials of new services and work processes, could enhance learning and care delivery. ${ }^{34}$

Under the broad category of experimentation, we include related learning processes such as improvisation, trial and error learning, learning from failures, refinement, exploitation, and exploration. We note, however, that some authors also distinguish experimentation as a separate learning process. ${ }^{21}$ The literature on learning in QI distinguishes deliberate experimentation from less intentional forms of experimentation like improvisation, as well as exploratory from exploitative forms of learning. Learning from failures also receives considerable attention.

\section{Deliberate experimentation versus improvisation}

Experimentation may be deliberate. Deliberate experimentation can promote collaboration and learning, but may cause an initial decrement in performance. ${ }^{23}$ Deliberate experimentation can also overcome potential pitfalls of superstitious learning (ie, fallacious causal assumptions based on previous, but limited experience). ${ }^{24}$ Experimentation can also increase diversity of experience that in turn may overcome obstacles to innovation adoption. ${ }^{30}$

Experimentation with new behaviors is a key part of processes for learning to use new technologies. ${ }^{25}$ Experimentation has also proved useful in determining how best to fulfill a legislative mandate to integrate shared decision making into clinical practice. ${ }^{32}$ Experimentation can also promote learning by refining an organization's overall approach to QI. ${ }^{26}$ 


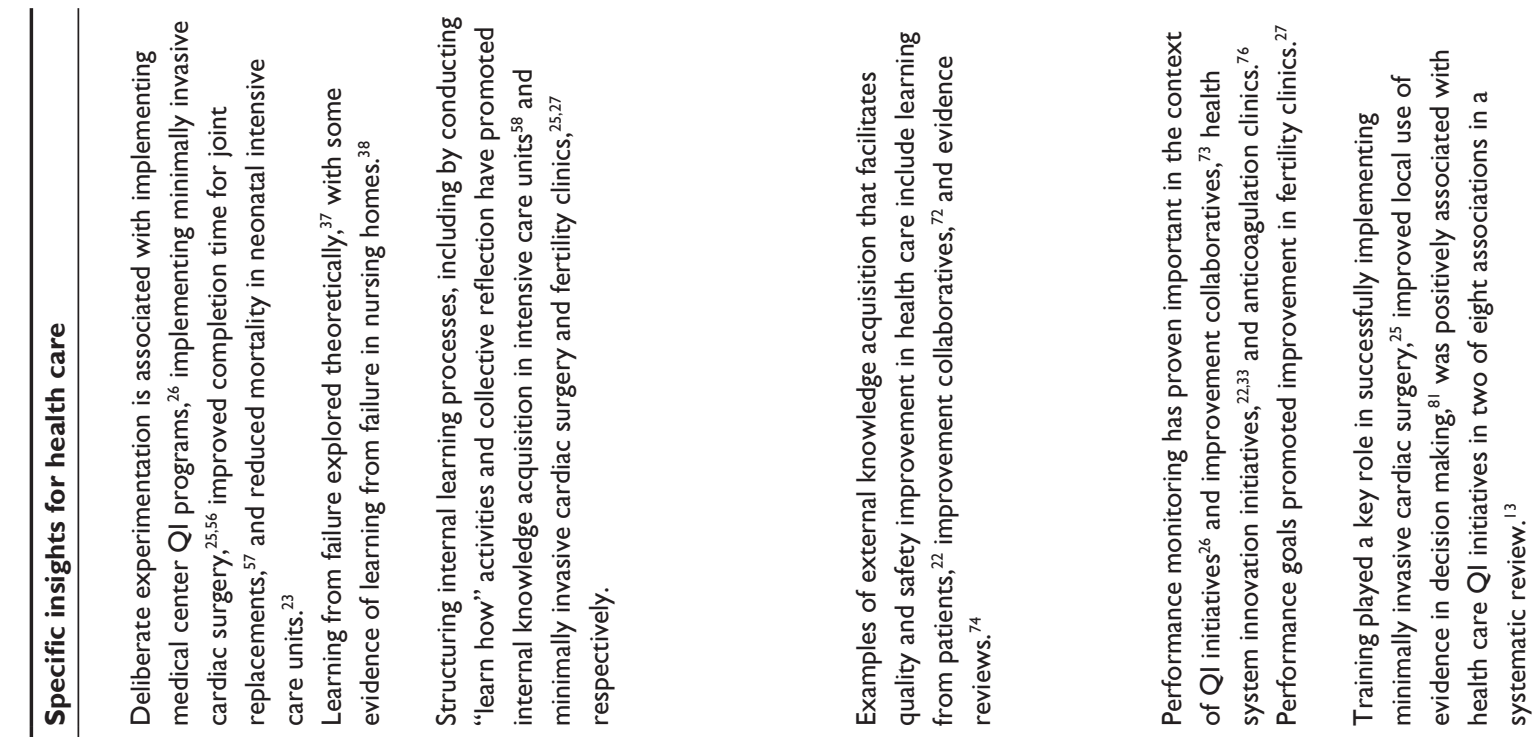

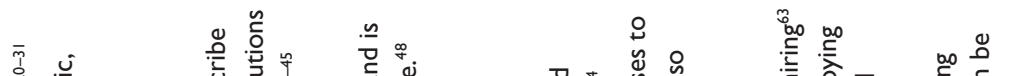

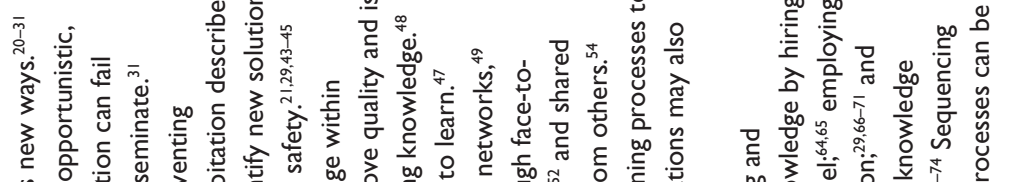

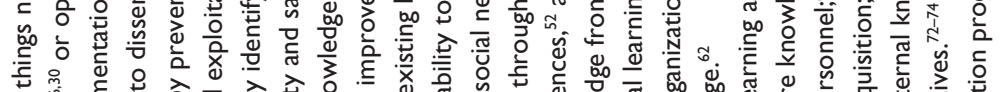

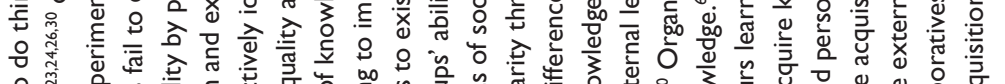

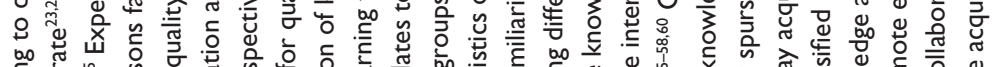

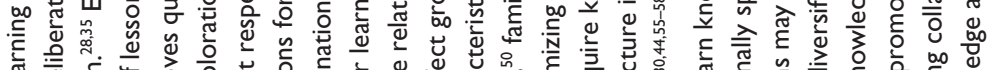

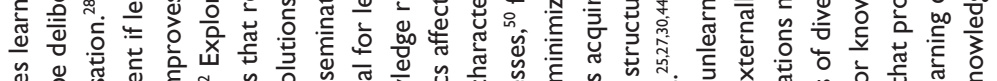

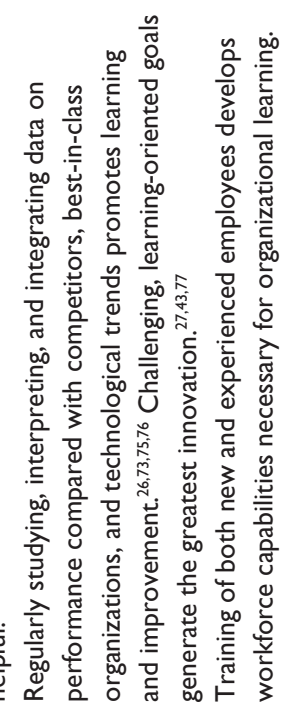

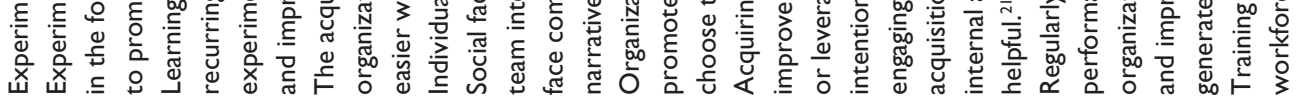
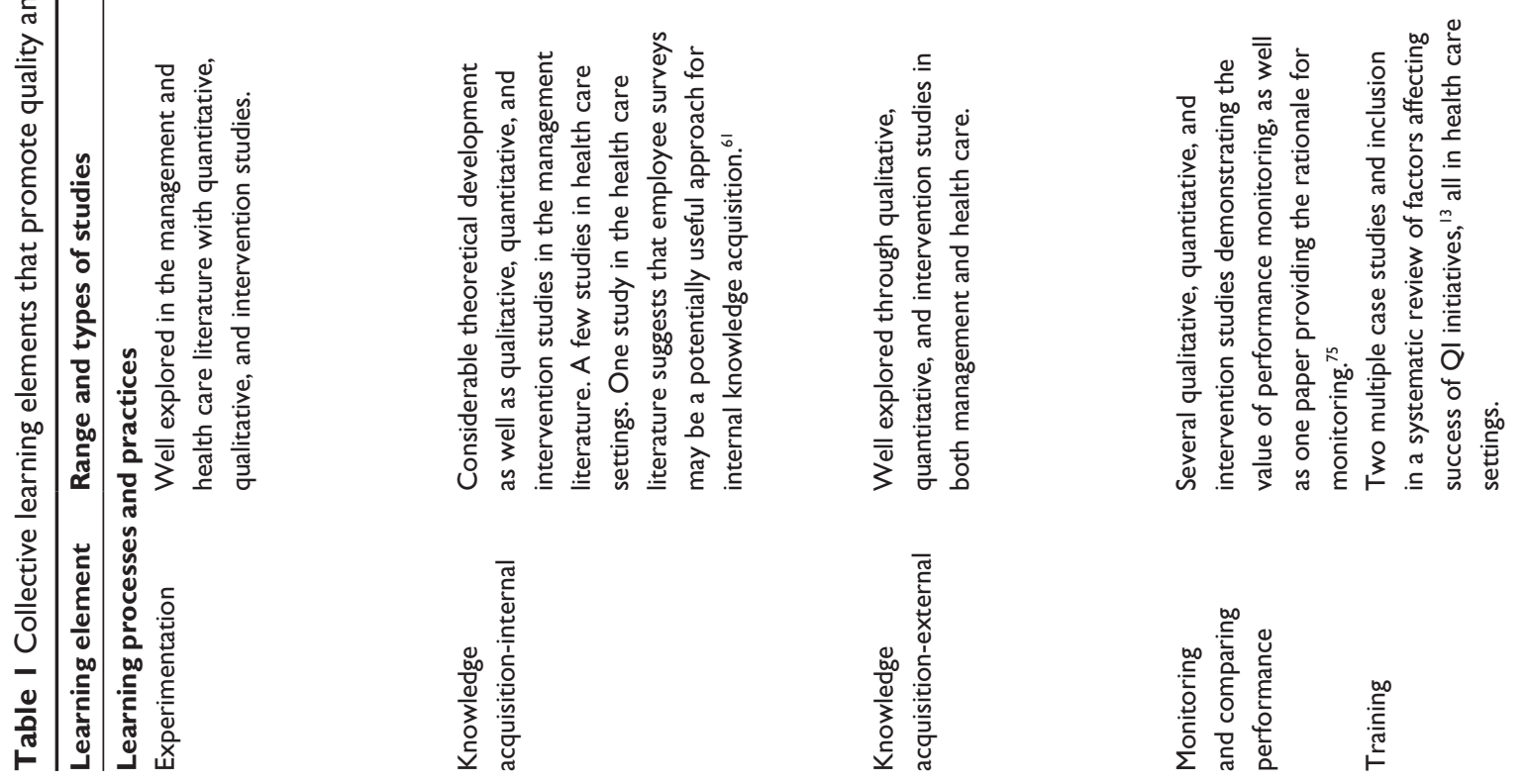

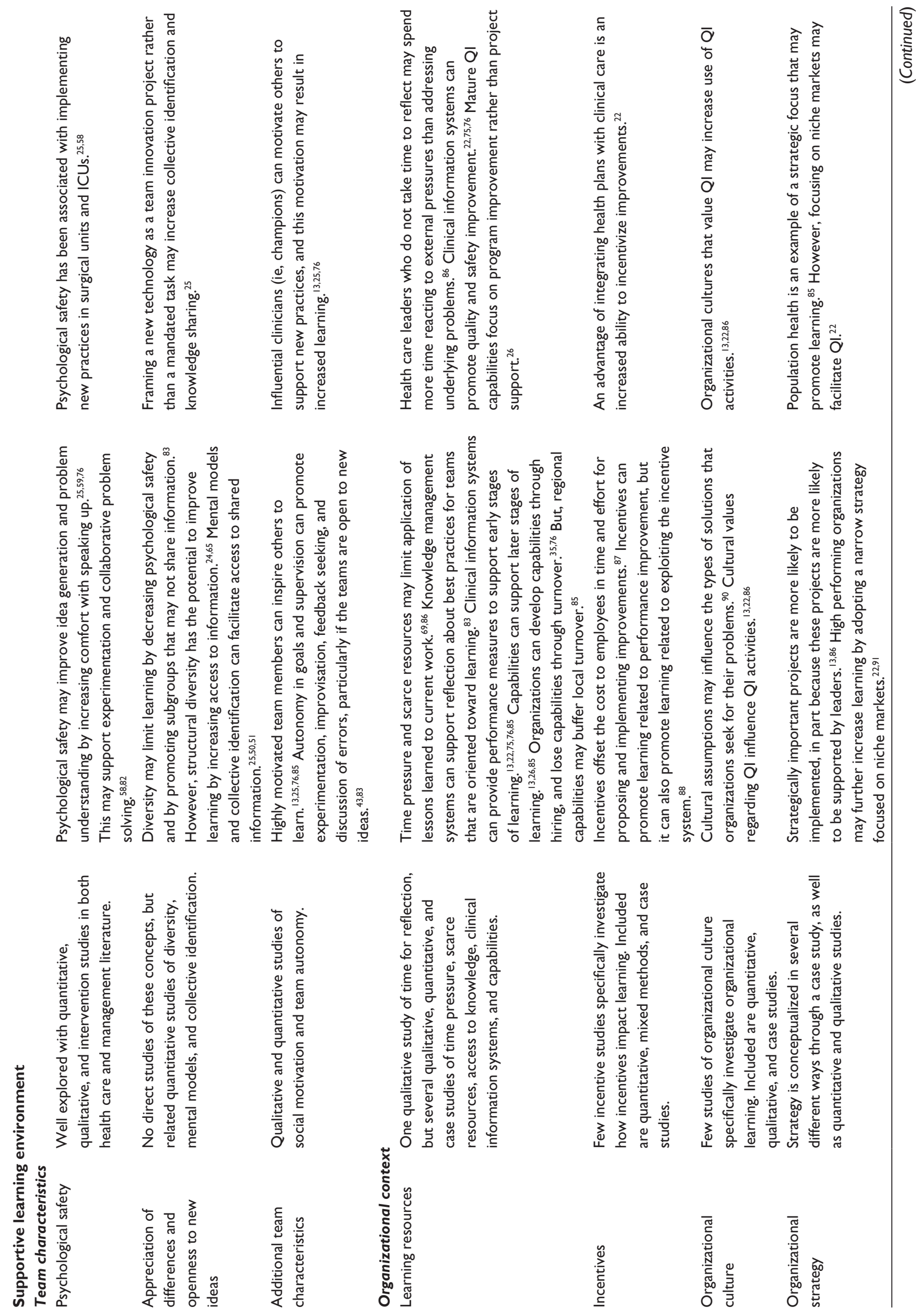

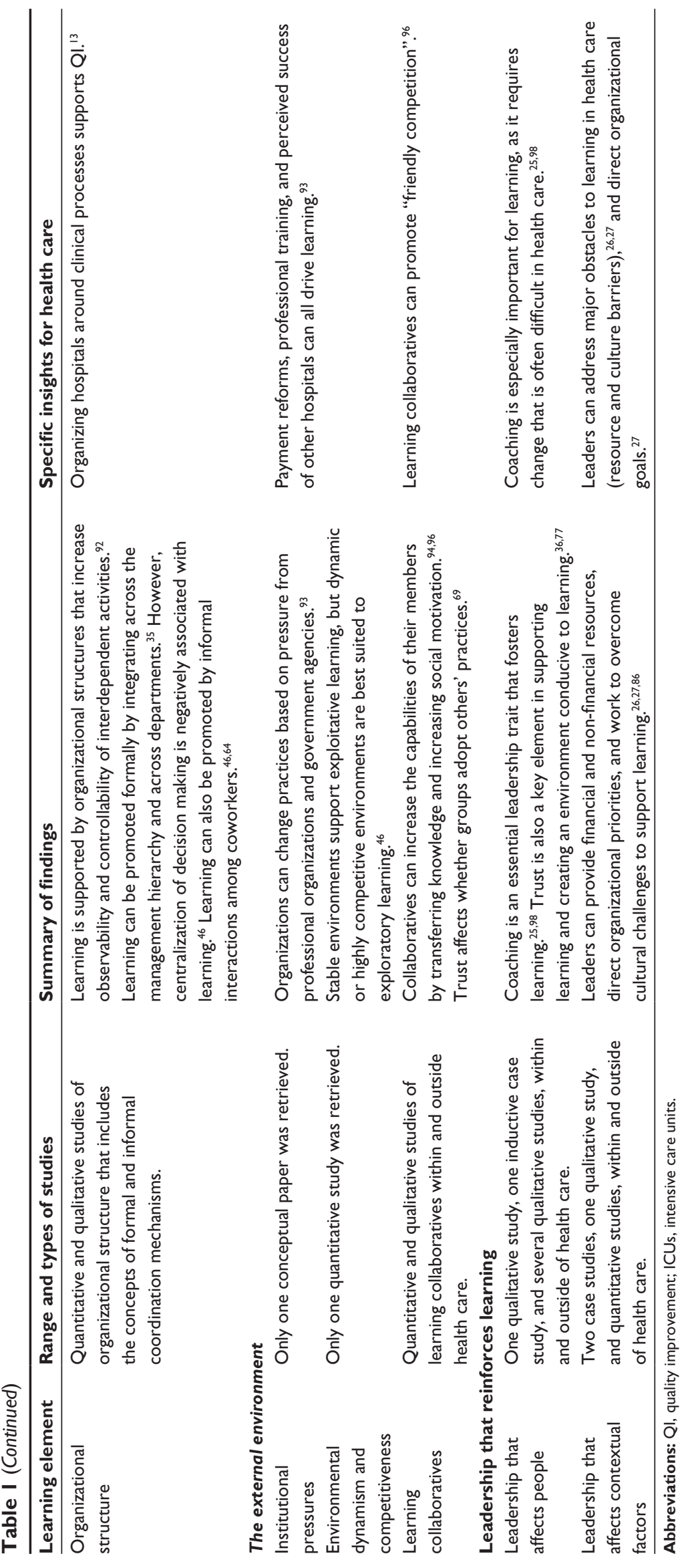
However, experimentation may not result in improvement if what has been learned locally fails to disseminate. ${ }^{31}$

Improvisation is similar to experimentation, but is opportunistic rather than deliberate. Organizations with improvisational competence can support long-term learning, ${ }^{28}$ and may affect large-scale change. ${ }^{35}$ However, improvisation may also interfere with learning by replacing deliberate experimentation. $^{28}$

\section{Learning from failures}

Learning from failures is a special form of experimentation that has received attention in the QI literature because it enables organizations to improve quality and safety in the longer term by preventing problems from recurring. ${ }^{36-42} \mathrm{One}$ health industry-focused article theorizes about factors that influence the ability of organizations to learn from failures, including characteristics of the adverse event; group composition, norms, safety management, as well as cultural, leadership, and network structures of organizations. ${ }^{37}$ The one empirical study of learning from failure in health care was set in nursing homes. ${ }^{38}$ It found that facilities learned from their own as well as others' failures, but learned less when they had a historical investment in the failing strategy. A related study characterizes trial and error learning as a process through which shared assumptions and values become linked to organizational routines over time. ${ }^{36}$ Thus, routines that are already heavily linked to shared assumptions and values may be difficult to change, even after failures.

\section{Exploration and exploitation}

Exploration and exploitation refer to characteristics of learning processes $;{ }^{21}$ respectively, they describe processes that are variance seeking versus mean seeking, ${ }^{43}$ or focused on acquiring new knowledge versus applying acquired knowledge.${ }^{29}$ Empirical studies have shown that exploratory and exploitative learning impact innovation and performance. One study found that exploratory and exploitative learning processes had complementary effects on innovation and performance in industrial firms. ${ }^{29}$ Others describe the value of sequencing between exploratory and exploitative learning, for example, in the case of replicating products or services. ${ }^{44}$ Research also demonstrates that exploitation can crowd out exploration. ${ }^{45}$

Exploration and exploitation occur through formal and informal coordination mechanisms, discussed further in the "The supportive learning environment" section on "Organization structure" below. In particular, informal social relations are helpful for both forms of learning. ${ }^{46}$

\section{Knowledge acquisition}

Knowledge acquisition is a process for obtaining and transmitting information in an organization. The literature on learning in QI elaborates the processes as well as structural and behavioral factors through which knowledge acquisition occurs. We note that some authors regard knowledge acquisition as a characteristic of exploration; ${ }^{29}$ here, we emphasize instead the experimental, variance seeking aspect of exploration and distinguish it from efforts to acquire knowledge. A major distinction in the literature and in practice is between internal and external knowledge acquisition. The difference involves drawing knowledge from an organization's own experience and expertise versus gaining knowledge from outside experts and customers of the organization. Research suggests that sequencing of internal and external knowledge acquisition, choosing which learning processes to use when, impacts short- and long-term learning. ${ }^{21}$

\section{Internal knowledge acquisition}

Internal knowledge acquisition depends on individual, social, and structural factors. An individual's intellectual demands and learning-orientation affect the extent to which knowledge acquisition from others occurs. ${ }^{47}$ Research suggests that the greatest learning occurs when new knowledge acquired is related to existing knowledge, rather than being specialized or totally unrelated to existing knowledge. ${ }^{48}$

Social and structural factors are described more fully in the "Supportive learning environment" section, below. However, a key concept is that social factors (eg, characteristics of social networks, ${ }^{49}$ managing team interaction [eg, communication and conflict resolution] processes,${ }^{50}$ familiarity through faceto-face communication, ${ }^{51}$ minimizing differences, ${ }^{52}$ shared narratives $)^{53}$ help groups encode, retrieve, and communicate knowledge stored by different individuals. ${ }^{54}$

Organizations can structure internal learning processes to promote improvement. This literature emphasizes how to structure experiential learning, both technical and organizational. ${ }^{30,55}$ Research reinforces the value of learning from experience, ${ }^{56}$ and demonstrates that team learning benefits not only from proficiency of individual workers and ability of workers to leverage other workers' knowledge, but also from the capacity of organizations to coordinate activity. ${ }^{57}$ Other ways to structure learning processes include conducting replication of practices in new settings; 44 "learn how" activities that operationalize practices in a given setting: ${ }^{.5}$ structured problem solving methods; ;9,60 employee surveys to assess learning culture; ${ }^{61}$ boundary crossing coordination practices that enable ongoing revision and alignment by making work 
visible to others; ${ }^{60}$ and using collective reflection processes to capture and embed new knowledge in the form of routines, technologies, and procedures. ${ }^{25,27}$ Organizations also need to actively work to consolidate or maintain knowledge - or conversely choose to abandon or unlearn knowledge. ${ }^{62}$

\section{External knowledge acquisition}

Acquiring knowledge from individuals and entities outside an organization can be a fruitful way for organizations to learn. Notable examples are learning from patients ${ }^{22}$ as well as the potential for identifying new knowledge by looking to other countries and across industries. ${ }^{33}$

External knowledge acquisition often occurs through processes involving people. For example, the literature describes the role of hiring individuals with specific skills or knowledge, ${ }^{63}$ leveraging network ties, ${ }^{64}$ system membership, and use of consultants; ${ }^{55}$ and diversifying work groups. ${ }^{65}$ External knowledge acquisition also occurs through a variety of systems-oriented processes. One notable strategy is mimicking the successful strategies of others. However, mimicking is hard, for three reasons: 1) successful strategies can be complex and thus resistant to logical, algorithmic efforts to imitate them; 2) incremental efforts to imitate successful strategies often encounter constraints that prevent complete imitation; and 3) mimicking entire strategies is difficult because small errors in imitated strategies can have large consequences. ${ }^{66}$ Further, when organizations successfully imitate an innovative organization, the innovator may also benefit because imitators generate knowledge that innovators can use to further innovate, particularly when the knowledge generated is familiar. ${ }^{67}$

Similar to mimicry, organizations can acquire knowledge by vicarious learning through collecting, codifying, and combining knowledge ${ }^{68}$ or through identifying, translating, adopting, and continuing knowledge. ${ }^{69}$ Organizations also acquire knowledge by creating absorptive capacity - the capability of organizations to utilize external knowledge for learning - in interfirm knowledge transfer. ${ }^{29}$ One paper examined the causal relationship between absorptive capacity, defined as output from research and development, and organizational investment in knowledge acquisition, in other words the study asked whether knowledge acquisition is "a chicken or an egg". Empirical evidence they present suggests that rather than building absorptive capacity to derive greater benefit from knowledge acquisition, some organizations have higher returns to knowledge acquisition and thus invest more. ${ }^{70}$ Finally, selecting carefully where to search for new knowledge, how long and how intensely to search, and ensuring fit between search location and intensity appears important in the success of knowledge acquisition processes. $^{71}$

QI collaboratives are a unique structural form that is increasingly used to promote knowledge sharing and acquisition across organizations. Learning collaboratives are networks of organizations or patient care units within organizations that work together to solve problems related to patient care quality or safety. Most evidence for QI collaboratives, albeit limited, is positive. ${ }^{72,73}$ Learning sessions have been described as a key element for shared knowledge acquisition within collaboratives. One study also found that conducting evidence reviews that are responsive to the needs of frontline innovators provides useful information in the context of a QI collaborative. ${ }^{74}$

\section{Monitoring and comparing performance}

Performance monitoring refers to learning to improve quality through a disciplined approach to studying and interpreting data, including comparisons with competitors, best-in-class organizations, and technological trends. Articles report and call for careful monitoring to evaluate workforce and other delivery system innovations. ${ }^{22,33}$ Learning to operate as a highly reliable organization requires integrating multiple sources and interpretations of data across several levels of analysis. ${ }^{75}$ Articles indicate that level and intensity of performance monitoring processes differentiated anticoagulation clinics, ${ }^{76} \mathrm{QI}$ collaboratives, ${ }^{73}$ and organizations implementing total quality management. ${ }^{26}$

Evaluating individual QI projects as well as an organization's improvement program as a whole entails second order learning. Second order learning derives from an assessment of not only whether QI is being done correctly but also whether the right QI activities are being done, for the right reasons. ${ }^{26}$ Identifying measurable outcomes and formulating challenging and achievable QI targets is not always straightforward, but plays a critical role in organizational learning. ${ }^{73}$

\section{Challenging performance goals}

A particular aspect of performance monitoring relates to performance targets. Choosing difficult goals is associated with higher performance, or more improvement, because doing so promotes spillovers, ie, opportunities to apply lessons learned under challenging circumstances more broadly, and thus faster learning. ${ }^{27}$ More nuanced research finds that, in research and development teams where creativity is key, learning-oriented goals related to both individual and team creativity, while performance-oriented goals did not. ${ }^{77}$ 
Similarly, in business development teams where exploratory learning was desirable, less directive goals achieved greater variance and thus more innovation. In contrast, when the best idea is known, more directed goals are helpful to promote learning that hones in on the best practice. ${ }^{43}$ Of particular concern, the Department of Veterans Affairs experience suggests that difficult performance goals may promote "gaming" rather than improved performance. ${ }^{78,79}$

\section{Training}

Training applies to both new and experienced employees. Training focuses on learning individual skills to develop workforce capabilities that are necessary for collective learning. For example, teams that succeeded in learning to use a new technology in cardiac surgery: 1) selected individuals for training based on having the skills needed to perform the work and the ability to train others, 2) conducted extensive training before performing the work through lectures, hands on simulation, and team-based dry runs to practice the procedure, 3) continued "learning by doing" through coaching team members during initial trials of the new technology, and 4) collectively reflected after, between, and during trials in order to inform subsequent trials. ${ }^{25}$

Supplemental training in QI, above and beyond the years of specialized training that health care professionals undertake before becoming eligible to practice their occupation, is often considered important to develop workforce capabilities. However, empirically, findings for a positive association of training programs with QI have been mixed. ${ }^{13}$ Case studies on training in the use of evidence-based processes suggest that training impacts local more than organizational learning. ${ }^{80}$

\section{The supportive learning environment}

Promoting organizational learning requires careful attention to the environment in which learning is intended to occur. The reviewed research lends to conceptualizing a supportive learning environment at three levels of analysis: team characteristics, organizational context of teams, and external environment.

\section{Team characteristics}

Team characteristics are features of teams or workgroups that are thought to influence organizational learning. Team characteristics include psychological safety, appreciation of differences, openness to new ideas, diversity, mental models, collective identification, social motivation, and team autonomy.

\section{Psychological safety}

Psychological safety benefits team learning by increasing members' comfort in asking questions and speaking up about concerns. Qualitative studies have found that comfort with speaking up, encouraging discussion, and seeking the opinions of others are associated with implementing new practices and higher quality of care. ${ }^{25,76}$ Psychological safety impacts quality and safety improvement by increasing idea generation and improved problem understanding. . $^{58,59}$ A mixed methods intervention study suggests that psychological safety promotes QI by stimulating collaboration and problem solving. ${ }^{81}$

\section{Appreciation of differences and openness to new ideas}

The reviewed studies did not specifically explore the role of appreciation of differences and openness to new ideas in quality and safety improvement. However, research on diversity, mental models, and collective identification each elaborate how and why these characteristics of work environments are important.

\section{Diversity}

Team learning may be strongest at low and high levels of team diversity. ${ }^{82}$ Teams with low diversity are better at communicating and experimenting, in part because members feel greater psychological safety to express opinions. Intermediate diversity is thought to inhibit learning because people tend to informally organize into subgroups that may inhibit knowledge sharing. High diversity limits subgroup formation because everybody is different and so members are more accepting of different viewpoints and experimentation.

Diversity is commonly conceived as differences in demographics, but differences in structural dimensions (eg, physical setting, training and experience, reporting managers) may impact organizational learning by increasing the diversity of social connections available to individuals and increasing knowledge by exposing members to different sources of task information, know-how, and feedback. ${ }^{65}$ Thus, organizations with heterogeneous experiences may be better able to identify best practices and make higher quality strategic decisions. ${ }^{24}$

\section{Mental models}

A key problem with diversity may be accessing knowledge distributed across team members. ${ }^{82}$ Teams can improve knowledge sharing and performance by developing mental models regarding who knows what. ${ }^{51}$ Team familiarity and 
face-to-face communication help teams develop these mental models. $^{51}$

\section{Collective identification}

Teams can also improve knowledge sharing by promoting collective identification to improvement projects because the commitment to team goals creates a common framework for the group to value others' contributions. ${ }^{50}$ Similarly, a study of surgical teams found that framing an improvement project as a team innovation rather than a mandated task impacted motivation. ${ }^{25}$ It is possible that this framing increased collective identification with the project.

\section{Additional team characteristics}

Social motivation and team autonomy were two additional team characteristics that help create a supportive learning environment.

\section{Social motivation}

A quality champion is known to improve motivation to change. ${ }^{13}$ In one study, influential clinicians promoted a sense of urgency to make use of clinical records to improve patient care. ${ }^{83}$ Another study showed that higher performing sites had an employee who was motivated to improve care. ${ }^{76}$ Broadly, social interactions may promote motivation by inspiring others to learn. For example, a study of surgical teams indicated that providing meaning by communicating the patient benefits of a new procedure increases learning for tedious procedures. ${ }^{25}$

\section{Team autonomy}

Team autonomy may impact learning by increasing discretion in goals and supervision. Autonomy in goals and supervision promotes experimentation, improvisation, feedback seeking, and discussion of errors when teams use exploratory learning, while less autonomy may be beneficial when teams use exploitative learning. ${ }^{43}$ Teams with very high or low team diversity have been shown to be unable to take advantage of autonomy, possibly because they are not prepared for different viewpoints or tolerant of experimentation. ${ }^{82}$

\section{Team context}

Team context comprises the characteristics of the broader organization that are thought to influence organizational learning through their intermediate impact on team functioning. Our review identified team context factors including learning resources, time for reflection, incentives, organizational culture, organization strategy, and organization structure.

\section{Learning resources}

Teams benefit from resources that enhance learning, including time for reflection, access to knowledge, and organizational capabilities.

\section{Time for reflection}

Deliberate reflection is important to support proactive and creative problem solving. ${ }^{84}$ Time pressure and scarce resources may influence continued use of a sub-optimal process by limiting searches for better alternatives. ${ }^{69}$ A qualitative study showed that high performing learning groups took the time to reflect collectively on events and information. Time for reflection allowed these groups to consider lessons learned, and apply those lessons to new problems. Lower performing groups struggled simply to find time to meet. These groups reacted to critical incidents and external pressures but did not take time to address underlying problems. ${ }^{84}$

\section{Access to knowledge}

Organizations can support learning through knowledge management systems (ie, databases for collecting and transferring best practices) and clinical information systems (ie, clinical data organized for quality and safety improvement). Knowledge management systems facilitate reflection on teams' current practices and lead to experimentation, but this depends on the degree to which the team is already oriented towards learning. ${ }^{82}$ Clinical information systems can facilitate QI activity. ${ }^{13,76}$ For example, Geisinger integrates key QI processes into the clinical information systems and tracks performance metrics. ${ }^{22}$ The Agency for Healthcare Research and Quality Patient Safety Indicators are another metric that could be used to support organizational learning for safety. ${ }^{75}$ Clinical information systems have been shown to facilitate change, even in the absence of strong social motivation. ${ }^{83}$ However, this study found that clinical information systems were important for the early years of organizational learning, while improvements to organizational capabilities were important for later years. ${ }^{83}$

\section{Organizational capabilities}

Organizational capabilities include the individual knowledge, skills, and abilities of individual employees, as well as practices, procedures, and policies. QI maturity is one capability that has been associated (11/14 studies) with implementation of QI activities. ${ }^{13} \mathrm{QI}$ maturity is conceptualized along several levels. ${ }^{26}$ Baseline QI maturity has been defined as creating an infrastructure (ie, hiring and training staff and engaging leaders). More mature QI programs develop and continuously improve clinical information systems, develop strong team 
characteristics, and directly involve executives in QI projects. Highly mature QI programs focus on whole systems rather than specific clinical processes.

Hiring strategies are important in ensuring that staff have the capabilities to engage in learning. For example, hiring practices may select practitioners who are more open to learning. ${ }^{35}$ Similarly, personnel turnover may negatively impact organizational capabilities. However, one study found that organizations with strong regional support systems were able to sustain performance despite staff turnover. ${ }^{83}$ Similarly, effective use of support staff can allow higher trained staff to better use their capabilities when staffing levels are low. ${ }^{76}$

\section{Incentives}

Health systems use incentives to promote learning. ${ }^{22}$ Incentives promote organizational learning by offsetting the cost to employees of time and effort related to proposing and implementing improvements. ${ }^{85}$ Incentives have been shown to promote "productive learning" regarding how to improve performance such as quality or safety in the short term..$^{85,86}$ However, incentives can also promote "adverse learning", ie, learning to exploit the system for personal benefit. In practice productive learning occurs in early stages of an incentive program, with adverse learning occurring later, after the most obvious improvements have been implemented. ${ }^{86}$

\section{Organizational culture}

Organizational culture is a pattern of assumptions that are developed over time as organizations learn how to solve key problems. ${ }^{87}$ For example, in the Geisinger Health System, a core assumption is that clinical system redesign is collaborative in that it involves clinical, operational, financial, payer, and patient stakeholders. ${ }^{22}$ The concept of a "strong" culture refers to assumptions that are widely shared and visible to outside observers. ${ }^{88}$ Strong cultures decrease performance variability and increase reliability over time. ${ }^{88}$ Organizational cultures that value QI in solving problems are likely to implement QI activities. ${ }^{13}$ Those who value QI invest time up front for improvements, rather than viewing QI as taking away from clinical time. ${ }^{84}$

\section{Organization strategy}

Implementation of QI activities is associated with the strategic importance of the project. ${ }^{13}$ In particular, strategic importance may facilitate collaboration among interdisciplinary teams of managers. ${ }^{84}$ Population health is one example of a broad strategic focus that is relevant for current United States health care reform. A population health strategy focuses an organization on promoting health more broadly than just those presenting for treatment. A population health orientation is one factor that may support sustained learning. ${ }^{83}$ However, organization theory suggests that strategies that narrowly focus on particular "products" that are associated with specific marketing niches may have advantages over more general strategies, particularly for already high performing organizations ${ }^{89}$ For example, Geisinger focuses innovations on the one third of patients for which the system is clinically and financially (through the health plan) responsible. ${ }^{22}$

\section{Organization structure}

Organizing hospitals around clinical processes (ie, clinical integration) can promote implementation of QI activities. ${ }^{13}$ One reason is that organizational learning may be easier when interdependent activities are more easily observable and controllable by managers. ${ }^{90}$ Coordination mechanisms are characteristics of the organization structure that can affect how and where learning occurs across an organization. Coordination can occur vertically (ie, across the management hierarchy) as well as horizontally (ie, across services or departments). Vertical coordination (ie, involvement of senior professionals in practices) can increase visibility of the daily challenges, local improvisations, and the need to coordinate these improvisations. ${ }^{35}$ Horizontal coordination (ie, practice-level professionals participating in overlapping teams) can increase both diffusion of improvisations and also legitimacy ascribed to the improvisations. ${ }^{35}$ Formal coordination mechanisms can vary in the degree of centralization and formalization. Centralization (ie, concentration of decision-making) may reduce the likelihood that employees will seek non-routine or innovative solutions to problems, relying instead on central guidance ${ }^{46}$ Formalization (ie, codified rules) may increase exploitative learning by prescribing practices used in incremental improvements. ${ }^{46}$ Informal coordination mechanisms are the interconnections among coworkers that form the basis for social relationships. Formal and informal coordination mechanisms affect exploration and exploitation differently. ${ }^{46}$ Specifically, both exploration and exploitation benefit from interpersonal connections within units. Centralized decision making limits exploration, whereas formalization can support exploitation.

\section{External environment}

The external environment includes factors that affect the health care industry or specific subsets of health care organizations. Factors highlighted by our review include institutional pressures, environmental dynamism and competitiveness, and learning collaboratives. 


\section{Institutional pressures}

One conceptual paper discusses how the health care industry receives coercive (ie, imposed on the industry), normative (ie, driven by assumptions regarding appropriate actions), or mimetic (ie, modeling organizations that appear successful) pressure to adopt certain practices. ${ }^{91}$ For example, the Affordable Care Act may provide coercive pressure by incentivizing quality and safety improvement. Professional training may provide normative pressure by increasing acceptance of professional values related to management control. The perceived success of hospitals that utilize quality and safety improvement programs could provide mimetic pressure by increasing visibility of QI.

\section{Environmental dynamism and competitiveness}

Dynamism (ie, instability) and competitiveness influence the effectiveness of exploratory and exploitative learning. ${ }^{46}$ Incremental improvements (ie, exploitation) are more effective in stable environments. Radical changes (ie, exploration) are more effective in environments where current practices become obsolete due to dynamism or competition.

\section{Learning collaboratives}

Experiences in learning collaboratives increase performance for members by providing opportunity and motivation to transfer knowledge. ${ }^{92}$ However, there are at least two challenges related to learning collaboratives. First, unlike research on other industries, ${ }^{92}$ individual health care organizations may not fully share the strategic goals of the learning collaborative in which they participate. Where organizational goals are clearly aligned with those of the collaborative, internal change is more likely. ${ }^{93}$ Second, collaborative members may have unequal access to information and communication that can limit members' ability to plan and draw on learning from across the collaborative. ${ }^{94}$

The literature identifies three potential ways that learning can be increased in learning collaboratives. First, collaboratives that emphasize "friendly competition" motivate organizational learning. ${ }^{94}$ Second, trust among collaborative members impacts learning by determining whether organizations will accept others' experience regarding new practices. ${ }^{69}$ Third, senior leaders increase the effectiveness of learning collaboratives by sharing power. A key finding from a study of product development collaboratives in the information technology industry was that collaboratives were most effective if they rotated leadership as the project evolved. ${ }^{95}$ Rotating leadership allowed the collaborative to better access the complementary capabilities of learning collaborative members as needed, and to broaden collective learning by exploring different aspects of problems based on different members' strategic priorities. Finally, effective collaboratives managed participation by identifying the appropriate experts in each organization for each project phase. These experts in turn involved others.

\section{Leadership that reinforces learning}

Leadership is critical for learning at every level from regional health leaders to managers within hospitals..$^{13,22,83}$ Leadership can impact learning both directly by impacting learning processes and practices and indirectly by developing a supportive learning environment. Leaders are essential in providing the guidance and direction necessary to directly champion and sustain learning, especially in the complex world of health care with established routines..$^{22,13}$ Leaders influence process and environment through actions and behaviors directed at people and contextual factors.

\section{Leadership that affects people}

Leaders support learning through coaching, by offering individuals and groups feedback about ways to improve specific processes and practices. ${ }^{25,96} \mathrm{~A}$ coaching approach also promotes psychological safety, increases expression of concerns and new ideas, and in turn improves the environment for learning. ${ }^{25,96}$ Research suggests that coaching promotes problem solving by developing a shared mental model between leaders and team members. Teams with managers who coach are more likely to share mental models of team performance with their manager, especially compared to managers who adopt a "blaming approach" ${ }^{96}$ Coaching by local leaders similarly played a critical role in enabling teams to learn to perform minimally invasive cardiac surgery. ${ }^{25}$ Surgeons in successful teams developed the capabilities of their teams by carefully selecting team members, and by supporting and participating in team-based training. Successful surgeons also encouraged social relationships across the operating room hierarchy, which allowed all members of the operating team to voice opinions and concerns. Coaching helped the surgeons change the organizational culture and create a supportive learning environment. Further, by recognizing the new procedure as a break from old procedures, surgeons were able to guide the changes necessary to successfully embed the new routine. ${ }^{25}$

Trust between leaders and workers strengthens connections between them. ${ }^{77}$ Research on both hospital-based QI programs and development of new practices suggests that strong connections between leaders and workers in terms of goals and perspectives, with hospital senior managers who are aware of and responsive to worker concerns, provide an environment that supports learning. ${ }^{26,36}$ Similarly, when 
implementing new practices, senior managers build trust by being aware of staff concerns in order to revise expectations. ${ }^{36}$ However, one study suggests limits to the beneficial effect of trust. That is, strong trust between supervisors and teams has the potential to promote complacency and acceptance of low quality outcomes. ${ }^{77}$ This research differentiated teams that focused on learning goals, ie, those that focus teams on better understanding the required tasks, from teams that focused on performance goals, ie, those that focus teams on achieving a certain level of performance. Both types of goals were shown to promote information exchange among team members. However, trust relationships with supervisors were shown to be beneficial for teams with learning goals, but decreased creativity for teams with performance goals. Researchers speculated that a trust relationship in teams that focus on performance goals may promote complacency and acceptance of low quality outcomes.

\section{Leadership that affects contextual factors}

Senior leaders are uniquely positioned to increase opportunities for learning by changing contextual factors. ${ }^{26,27,84}$ Leaders provide the resources needed to support learning, including financial and non-financial incentives, electronic health records, staffing for QI projects, and quality and outcome measures. ${ }^{22,83,84}$ Leaders support learning by emphasizing a culture of respect and openness and displaying a willingness to proactively solve problems. ${ }^{84}$ They also take strategic action to support a learning environment by increasing opportunities or reducing barriers to learning and addressing cultural challenges ${ }^{26,27}$ For example, British in vitro fertilization clinics that served more challenging patients learned faster than clinics that undertook less challenging patients and over time surpassed their performance in terms of live births. ${ }^{27}$

Senior leaders impact the context of teams by emphasizing the strategic importance of learning. For example, one study found that government mandates to recall products resulted in lower learning than voluntary recalls. ${ }^{97}$ The authors suggest that decisions by managers to recall products signal the strategic importance of the recall to lower level managers and staff.

\section{Discussion}

\section{Extended theory of organizational learning}

In this review, we distilled research from leading health care and management journals that linked quality and safety improvement with collective learning into a framework that describes collective learning in terms of three building blocks: learning processes and practices, a supportive learning environment, and leadership that reinforces learning. Our findings confirm that collective learning plays a role in improving quality and safety in health care. Our review also allows us to identify ways to extend the original conceptual model in order to elucidate how each of the three building blocks support learning for quality and safety improvement (see Figure 1).

The original conceptual model identified experimentation, knowledge acquisition, performance monitoring, and training as key processes and practices that promote collective learning. Our review supports this classification and allows us to elaborate these categories in the context of quality and safety improvement. We find that experimentation includes a variety of related processes, including deliberate experimentation, improvisation, learning from failures, exploration, and exploitation. Knowledge acquisition can be either internal or external and both entail attention to individual, social, and structural factors, as well as learning from experience - a special form of self-learning. For quality and safety improvement, establishing challenging performance goals leverages efforts to monitor and compare performance.

To support these processes and practices, the original conceptual framework suggests the need for a supportive learning environment that includes psychological safety, appreciation of differences, openness to new ideas, and time for reflection. Our review enabled substantial elaboration of this learning block. Specifically, we classified aspects of a supportive learning environment as team characteristics, team contextual factors, and external environmental factors. In the original framework, psychological safety, appreciation of differences, and openness to new ideas are team characteristics. We also identified social motivation and team autonomy as important additional team characteristics. In addition, we found few articles that discussed the role of appreciation of differences and openness to new ideas directly in the context of quality and safety improvement initiatives. Rather, these tended to be desirable byproducts of team diversity, shared mental models, and collective identification.

In addition to these characteristics of teams, our review identified team contextual factors - learning resources, including time for reflection, access to knowledge, organizational capabilities; incentives; and organizational culture, strategy, and structure - that supported learning for quality and safety improvement. Of these, time for reflection was the only contextual factor identified in the original conceptual model. Similarly, the original conceptual framework did not specifically highlight how the external environment may influence organizational 


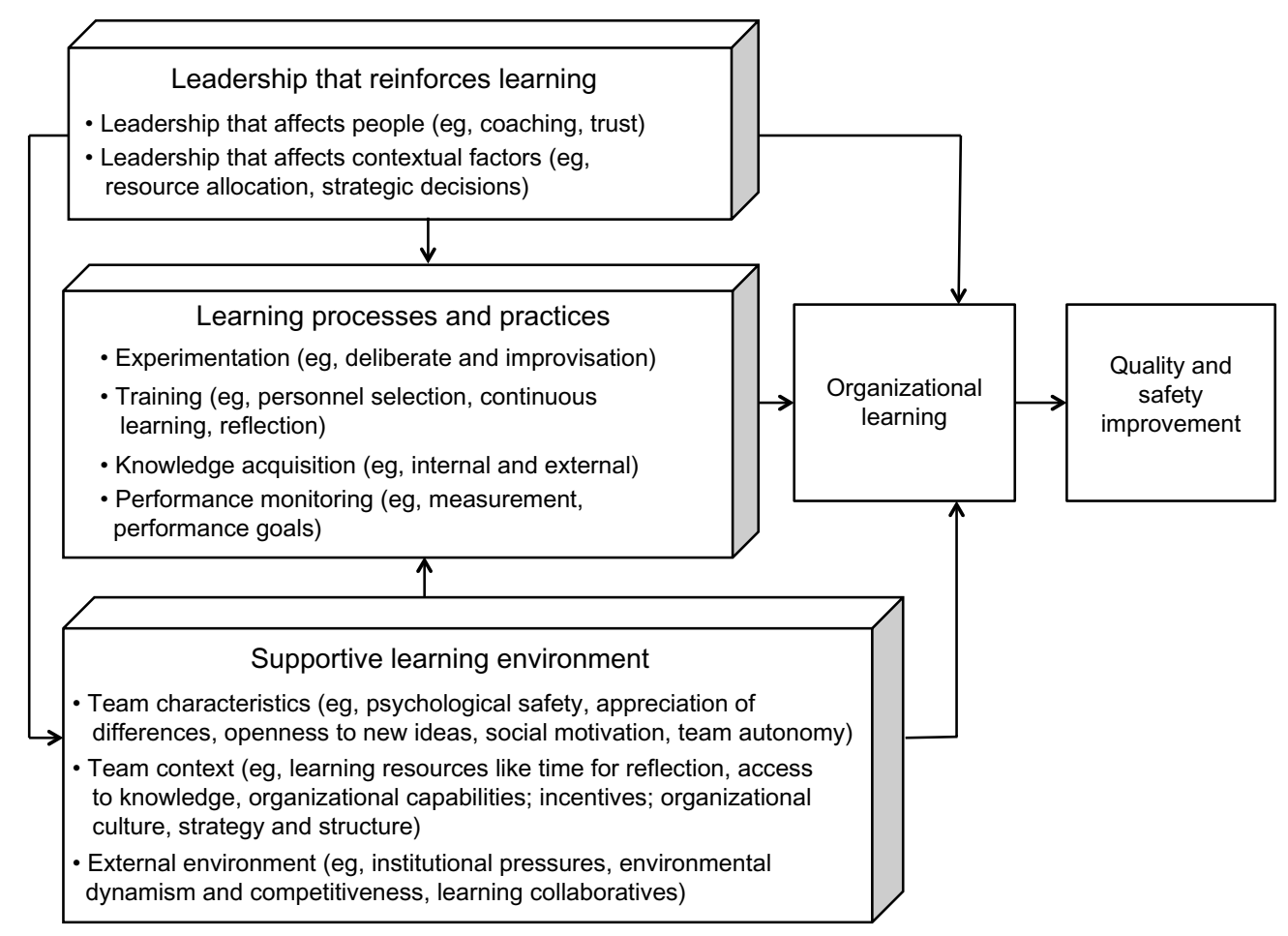

Figure I Conceptual model: how learning impacts quality and safety improvement.

Note: Copyright (C) 20I2 SAGE Publications. Adapted from Singer SJ, Moore SC, Meterko M, Williams S. Development of a short-form Learning Organization Survey: the LOS-27. Medical Care Research and Review. 2012;69(4):432-459. ${ }^{20}$

learning. This review adds to the conceptual framework by considering the role of institutional pressures, environmental dynamism and competitiveness, and learning collaboratives for organizational learning in the context of quality and safety improvement.

Recognizing that leaders play a key role in creating and maintaining the supportive learning environment and establishing learning processes and practices, the last building block is leadership that reinforces learning. Our review confirms that leadership is important for promoting collective learning in the context of quality and safety improvement. Specifically, our review highlights that leaders contribute through actions and behaviors that affect people, such as coaching and trust building, and through influencing contextual factors, including providing resources, developing culture, and taking strategic actions that support improvement.

\section{Limitations}

This review is limited by its methodological approach. As a scoping review, we considered only articles published in top tier and certain specialized journals in the health care and management literature. While this methodology facilitates a relatively quick elaboration of the literature by identifying the most relevant concepts, it does not allow complete coverage of topics studied or conclusive identification of research gaps. For example, this review finds few examples of incentives in relation to organizational learning in quality and safety improvement; yet we are aware of a large amount of literature on incentives that could arguably be integrated into our conceptual framework for collective learning. ${ }^{98-100}$ While the resultant conceptual model is substantially more articulated than its predecessor, it may still be incomplete. We thus recommend that it be viewed as a work in progress.

Despite its methodological limitations this review highlights the paucity of empirical research on collective learning in health care $(n=27)$ relative to the number of articles in management journals drawn from other industries $(n=49)$. This suggests a significant need for additional research on learning in health care quality and safety improvement, particularly regarding some areas of the conceptual model (see Table 1). For instance, our review highlights the importance of leadership in both promoting a supportive learning environment and implementing learning processes (ie, experimentation, knowledge acquisition, performance monitoring, and training). Researchers could productively focus on one of the four learning processes in order to determine how and under what conditions leaders are able to influence learning processes directly, as well as indirectly by developing a supportive learning environment. 


\section{Conclusion}

Challenged by urgent quality and safety problems in health care, health care leaders need guidance regarding how to achieve improvement. Collective learning may be key. This review summarizes research that identifies pathways toward improvement through learning, offering a comprehensive framework for strengthening health care delivery and ultimately saving lives.

\section{Disclosure}

The authors report no conflicts of interest in this work.

\section{References}

1. Committee on Quality of Health Care in America and Institute of Medicine. Crossing the Quality Chasm: A New Health System for the 21st Century. Washington: National Academies Press; 2001.

2. Classen DC, Resar R, Griffin F, et al. 'Global trigger tool' shows that adverse events in hospitals may be ten times greater than previously measured. Health Aff (Millwood). 2011;30(4):581-589.

3. McGlynn EA, Asch SM, Adams J, et al. The quality of health care delivered to adults in the United States. N Engl J Med. 2003;348(26):2635-2645.

4. Song Y, Skinner J, Bynum J, Sutherland J, Wennberg JE, Fisher ES Regional variations in diagnostic practices. NEngl J Med. 2010; 363(1): 45-53.

5. Birkmeyer JD, Reames BN, McCulloch P, Carr AJ, Campbell WB, Wennberg JE. Understanding of regional variation in the use of surgery. Lancet. 2013;382(9898):1121-1129.

6. Nelson AR, Smedley BD, Stith AY. Unequal Treatment: Confronting Racial and Ethnic Disparities in Health Care (full printed version). National Academies Press; 2002.

7. Newyorker.com [homepage on the Internet]. Overkill: An avalanche of unnecessary medical care is harming patients physically and financially. What can we do about it? Gawande A; 2015. Available from: http://www. newyorker.com/magazine/2015/05/11/overkill-atul-gawande. Accessed August 15, 2015

8. Committee on the Learning Health Care System in America; Institute of Medicine; Smith M, et al, editors. Best Care at Lower Cost: The Path to Continuously Learning Health Care in America. Washington: National Academies Press; 2013.

9. Bradley EH, Herrin J, Wang Y, et al. Strategies for reducing the door-to-balloon time in acute myocardial infarction. $N$ Engl J Med. 2006;355(22):2308-2320.

10. Pronovost $\mathrm{P}$, Needham D, Berenholtz S, et al. An intervention to decrease catheter-related bloodstream infections in the ICU. $N$ Engl J Med. 2006;355(26):2725-2732.

11. Nicolay CR, Purkayastha S, Greenhalgh A, et al. Systematic review of the application of quality improvement methodologies from the manufacturing industry to surgical healthcare. Br J Surg. 2012;99(3): 324-335.

12. Alexander JA, Hearld LR. The science of quality improvement implementation: developing capacity to make a difference. Med Care. 2011;49 Suppl:S6-S20.

13. Kaplan HC, Brady PW, Dritz MC, et al. The influence of context on quality improvement success in health care: a systematic review of the literature. Milbank Q. 2010;88(4):500-559.

14. Glasgow JM, Scott-Caziewell JR, Kaboli PJ. Guiding inpatient quality improvement: a systematic review of Lean and Six Sigma. Jt Comm J Qual Patient Saf. 2010;36(12):533-540.

15. Edmondson A. Psychological safety and learning behavior in work teams. Adm Sci Q. 1999;44(2):350-383.

16. Levitt B, March JG. Organizational learning. Annual Review of Sociology. 1988;14:319-340.
17. Crossan MM, Lane HW, White RE. An organizational learning framework: From intuition to institution. Academy of Management Review. 1999;24(3):522-537.

18. Arksey H, O’Malley L. Scoping studies: towards a methodological framework. International Journal of Social Research Methodology. 2005;8(1):19-32.

19. Levac D, Colquhoun H, O'Brien KK. Scoping studies: advancing the methodology. Implement Sci. 2010;5:69.

20. Singer SJ, Moore SC, Meterko M, Williams S. Development of a shortform Learning Organization Survey: the LOS-27. Med Care Res Rev. 2012;69(4):432-459.

21. Bingham CB, Davis JP. Learning Sequences: Their Existence, Effect, and Evolution. Acad Manage J. 2012;55(3): 611-641.

22. Paulus RA, Davis K, Steele GD. Continuous innovation in health care: implications of the Geisinger experience. Health Aff (Millwood). 2008;27(5):1235-1245.

23. Nembhard IM, Tucker AL. Deliberate Learning to Improve Performance in Dynamic Service Settings: Evidence from Hospital Intensive Care Units. Organization Science. 2011;22(4):907-922.

24. Zollo M. Superstitious Learning with Rare Strategic Decisions: Theory and Evidence from Corporate Acquisitions. Organization Science. 2009;20(5):894-908.

25. Edmondson AC, Bohmer RM, Pisano GP. Disrupted Routines: Team Learning and New Technology Implementation in Hospitals. Adm Sci Q. 2001;46(4):685-716.

26. Rivard PE, Parker VA, Rosen AK. Quality improvement for patient safety: project-level versus program-level learning. Health Care Manage Rev. 2013;38(1):40-50.

27. Stan M, Vermeulen F. Selection at the Gate: Difficult Cases, Spillovers, and Organizational Learning. Organization Science. 2013;24(3): 796-812.

28. Miner AS, Bassoff $P$, Moorman C. Organizational Improvisation and Learning: A Field Study. Adm Sci Q. 2001;46(2):304-337.

29. Lichtenthaler U. Absorptive capacity, environmental turbulence, and the complementarity of organizational learning processes. Acad Manage J. 2009;52(4):822-846.

30. Weigelt C, Sarkar MB. Learning from supply-side agents: the impact of technology solution providers' experiential diversity on clients' innovation adoption. Acad Manage J. 2009;52(1):37-60.

31. Lapré MA, Mukherjee AS, van Wassenhove LN. Behind the Learning Curve: Linking Learning Activities to Waste Reduction. Management Science. 2000;46(5):597.

32. King J, Moulton B. Group Health's participation in a shared decisionmaking demonstration yielded lessons, such as role of culture change. Health Aff (Millwood). 2013;32(2):294-302.

33. Macdonnell M, Darzi A. A key to slower health spending growth worldwide will be unlocking innovation to reduce the labor-intensity of care. Health Aff (Millwood). 2013;32(4):653-660.

34. Weaver SJ, Dy SM, Rosen MA. Team-training in healthcare: a narrative synthesis of the literature. BMJ Qual Saf. 2014;23(5):359-372.

35. Smets M, Morris TIM, Greenwood R. From practice to field: a multilevel model of practice-driven institutional change. Acad Manage J. 2012;55(4):877-904.

36. Rerup C, Feldman MS. Routines as a source of change in organizational schemata: the role of trial-and-error learning. Acad Manage J. 2011;54(3):577-610.

37. Chuang YT, Ginsburg L, Berta WB. Learning from preventable adverse events in health care organizations: development of a multilevel model of learning and propositions. Health Care Manage Rev. 2007;32(4): 330-340.

38. Chuang YT, Baum JAC. It's All in the Name: Failure-Induced Learning by Multiunit Chains. Adm Sci Q. 2003;48(1): 33-59.

39. Haunschild PR, Sullivan BN. Learning from Complexity: Effects of Prior Accidents and Incidents on Airlines' Learning. Adm Sci $Q$ 2002;47(4):609-643.

40. Henderson AD, Stern I. Selection-based Learning: The Coevolution of Internal and External Selection in High-velocity Environments. Adm Sci Q. 2004;49(1):39-75. 
41. Madsen PM. These Lives Will Not Be Lost in Vain: Organizational Learning from Disaster in US Coal Mining. Organization Science. 2009;20(5):861-875.

42. Madsen PM, Desai V. Failing to learn? The effects of failure and success on organizational learning in the global orbital launch vehicle industry. Acad Manage J. 2010;53(3):451-476.

43. McGrath RG. Exploratory learning, innovative capacity, and managerial oversight. Acad Manage J. 2001;44(1):118-131.

44. Winter SG, Szulanski G. Replication as Strategy. Organization Science. 2001;12(6):730-743.

45. Benner MJ, Tushman M. Process Management and Technological Innovation: A Longitudinal Study of the Photography and Paint Industries. Adm Sci Q. 2002;47(4):676-706.

46. Jansen JJ, Van Den Bosch FAJ, Volberda HW. Exploratory Innovation, Exploitative Innovation, and Performance: Effects of Organizational Antecedents and Environmental Moderators. Management Science. 2006;52(11):1661-1674.

47. Gray PH, Meister DB. Knowledge Sourcing Effectiveness. Management Science. 2004;50(6):821-834.

48. Schilling MA, Vidal P, Ployhart RE, Marangoni A. Learning by Doing Something Else: Variation, Relatedness, and the Learning Curve. Management Science. 2003;49(1):39-56.

49. Rulke DL, Galaskiewicz J. Distribution of Knowledge, Group Network Structure, and Group Performance. Management Science. 2000; 46(5):612.

50. Van Der Vegt GS, Bunderson JS. Learning and performance in multidisciplinary teams: the importance of collective team identification. Acad Manage J. 2005;48(3):532-547.

51. Lewis K. Knowledge and Performance in Knowledge-Worker Teams: A Longitudinal Study of Transactive Memory Systems. Management Science. 2004;50(11):1519-1533.

52. Majchrzak A, More PH, Faraj S. Transcending Knowledge Differences in Cross-Functional Teams. Organization Science. 2012;23(4): 951-970.

53. Garud R, Dunbar RLM, Bartel CA. Dealing with Unusual Experiences: A Narrative Perspective on Organizational Learning. Organization Science. 2011;22(3):587-601.

54. Lewis K, Lange D, Gillis L. Transactive Memory Systems, Learning, and Learning Transfer. Organization Science. 2005;16(6):581-598.

55. Walston SL, Kimberly JR, Burns LR. Institutional and economic influences on the adoption and extensiveness of managerial innovation in hospitals: The case of reengineering. Med Care Res Rev. 2001;58(2):194-228; discussion 229-133.

56. Pisano GP, Bohmer RM, Edmondson AC. Organizational Differences in Rates of Learning: Evidence from the Adoption of Minimally Invasive Cardiac Surgery. Management Science. 2001;47(6):752-768.

57. Reagans R, Argote L, Brooks D. Individual Experience and Experience Working Together: Predicting Learning Rates from Knowing Who Knows What and Knowing How to Work Together. Management Science. 2005;51(6):869-881.

58. Tucker AL, Nembhard IM, Edmondson AC. Implementing New Practices: An Empirical Study of Organizational Learning in Hospital Intensive Care Units. Management Science. 2007;53(6):894-907.

59. Choo AS, Linderman KW, Schroeder RG. Method and Psychological Effects on Learning Behaviors and Knowledge Creation in Quality Improvement Projects. Management Science. 2007;53(3):437-450.

60. Kellogg KC, Orlikowski WJ, Yates J. Life in the Trading Zone: Structuring Coordination Across Boundaries in Postbureaucratic Organizations. Organization Science. 2006;17(1):22-44.

61. Ohman-Strickland PA, John Orzano A, Nutting PA, et al. Measuring organizational attributes of primary care practices: development of a new instrument. Health Serv Res. 2007;42(3 Pt 1):1257-1273.

62. de Holan PM, Phillips N. Remembrance of Things Past? The Dynamics of Organizational Forgetting. Management Science. 2004;50(11): 603-1613.

63. Song J, Almeida P, Wu G. Learning-by-Hiring: When Is Mobility More Likely to Facilitate Interfirm Knowledge Transfer? Management Science. 2003;49(4):351-365.
64. Reagans R, McEvily B. Network Structure and Knowledge Transfer: The Effects of Cohesion and Range. Adm Sci Q. 2003;48(2):240-267.

65. Cummings JN. Work Groups, Structural Diversity, and Knowledge Sharing in a Global Organization. Management Science. 2004;50(3): 352-364.

66. Rivkin JW. Imitation of Complex Strategies. Management Science. 2000;46(6):824.

67. Yang H, Phelps C, Steensma HK. Learning from what others have learned from you: the effects of knowledge spillovers on originating firms. Acad Manage J. 2010;53(2):371-389.

68. Schulz M. The uncertain relevance of newness: organizational learning and knowledge flows. Acad Manage J. 2001; 44(4):661-681.

69. Bresman H. Changing routines: a process model of vicarious group learning in pharmaceutical R\&D. Acad Manage J. 2013; 56(1):35-61.

70. Knott AM. R\&D/Returns Causality: Absorptive Capacity or Organizational IQ. Management Science. 2008;54(12):2054-2067.

71. Li Q, Maggitti PG, Smith KG, Tesluk PE, Katila R. Top management attention to innovation: the role of search selection and intensity in new product introductions. Acad Manage J. 2013; 56(3):893-916.

72. Schouten LM, Hulscher ME, van Everdingen JJ, Huijsman R, Grol RP. Evidence for the impact of quality improvement collaboratives: systematic review. BMJ. 2008;336(7659):1491-1494.

73. Strating MM, Nieboer AP, Zuiderent-Jerak T, Bal RA. Creating effective quality-improvement collaboratives: a multiple case study. $B M J$ Qual Saf. 2011;20(4):344-350.

74. Danz MS, Hempel S, Lim YW, et al. Incorporating evidence review into quality improvement: meeting the needs of innovators. BMJ Qual Saf. 2013;22(11):931-939.

75. Rivard PE, Rosen AK, Carroll JS. Enhancing patient safety through organizational learning: Are patient safety indicators a step in the right direction? Health Serv Res. 2006;41(4 Pt 2):1633-1653.

76. Rose AJ, Petrakis BA, Callahan P, et al. Organizational characteristics of high- and low-performing anticoagulation clinics in the Veterans Health Administration. Health Serv Res. 2012;47(4):1541-1560.

77. Gong Y, Kim TY, Lee DR, Zhu J. A multilevel model of team goal orientation, information exchange, and creativity. Acad Manage $J$. 2013;56(3):827-851.

78. Kizer KW, Jha AK. Restoring trust in VA health care. $N$ Engl J Med. 2014;371(4):295-297.

79. Benzer JK, Creech SK, Mohr DC, Charns MP. Learning goals may prevent "goals gone wild". Am J Public Health. 2014;104(12):e1.

80. Champagne F, Lemieux-Charles L, Duranceau MF, MacKean G, Reay T. Organizational impact of evidence-informed decision making training initiatives: a case study comparison of two approaches. Implement Sci. 2014;9:53.

81. Bernstein ES. The Transparency Paradox: A Role for Privacy in Organizational Learning and Operational Control. Adm Sci Q. 2012;57(2):181-216.

82. Gibson C, Vermeulen F. A Healthy Divide: Subgroups as a Stimulus for Team Learning Behavior. Adm Sci Q. 2003;48(2):202-239.

83. Schierhout G, Hains J, Si D, et al. Evaluating the effectiveness of a multifaceted, multilevel continuous quality improvement program in primary health care: developing a realist theory of change. Implement Sci. 2013;8:119.

84. Singer SJ, Hayes JE, Gray GC, Kiang MV. Making time for learningoriented leadership in multidisciplinary hospital management groups. Health Care Manage Rev. Epub 2014 Jul 15.

85. Arthur JB, Huntley CL. Ramping Up the Organizational Learning Curve: Assessing the Impact of Deliberate Learning on Organizational Performance under Gainsharing. Acad Manage J. 2005;48(6):1159-1170.

86. Obloj T, Sengul M. Incentive Life-cycles: Learning and the Division of Value in Firms. Adm Sci Q. 2012;57(2): 305-347.

87. Schneider B, Ehrhart MG, Macey WH. Organizational climate and culture. Annual Review of Psychology. 2013;64:361-388.

88. Sørensen JB. The Strength of Corporate Culture and the Reliability of Firm Performance. Adm Sci Q. 2002;47(1): 70-91. 
89. Lapré MA, Tsikriktsis N. Organizational Learning Curves for Customer Dissatisfaction: Heterogeneity Across Airlines. Management Science. 2006;52(3):352-366

90. Sorenson O. Interdependence and Adaptability: Organizational Learning and the Long-Term Effect of Integration. Management Science. 2003;49(4):446-463.

91. Yang CW, Fang SC, Huang WM. Isomorphic pressures, institutional strategies, and knowledge creation in the health care sector. Health Care Manage Rev. 2007;32(3):263-270.

92. Ingram P, Simons T. The Transfer of Experience in Groups of Organizations: Implications for Performance and Competition. Management Science. 2002;48(12):1517-1533.

93. Hearld LR, Alexander JA, Mittler JN. Fostering change within organizational participants of multisectoral health care alliances. Health Care Manage Rev. 2012;37(3):267-279.

94. Dainty KN, Scales DC, Sinuff T, Zwarenstein M. Competition in collaborative clothing: a qualitative case study of influences on collaborative quality improvement in the ICU. BMJ Qual Saf. 2013;22(4): $317-323$
95. Davis JP, Eisenhardt KM. Rotating Leadership and Collaborative Innovation: Recombination Processes in Symbiotic Relationships. Adm Sci Q. 2011;56(2):159-201.

96. Buljac-Samardzic M, van Woerkom M, Paauwe J. Team safety and innovation by learning from errors in long-term care settings. Health Care Manage Rev. 2012;37(3):280-291.

97. Haunschild PR, Rhee M. The Role of Volition in Organizational Learning: The Case of Automotive Product Recalls. Management Science. 2004;50(11):1545-1560.

98. Rosenthal MB, Frank RG, Li Z, Epstein AM. Early experience with pay-for-performance: from concept to practice. JAMA. 2005;294(14): 1788-1793.

99. Christianson JB, Leatherman S, Sutherland K. Lessons from evaluations of purchaser pay-for-performance programs a review of the evidence. Med Care Res Rev. 2008;65(6 Suppl):5S-35S.

100. Benzer JK, Young GJ, Burgess JF Jr, et al. Sustainability of quality improvement following removal of pay-for-performance incentives. J Gen Intern Med. 2014;29(1):127-132.
Journal of Healthcare Leadership

\section{Publish your work in this journal}

The Journal of Healthcare Leadership is an international, peer-reviewed, open access journal focusing on leadership for the health profession. The journal is committed to the rapid publication of research focusing on but not limited to: Healthcare policy and law; Theoretical and practical aspects healthcare delivery; Interactions between healthcare and society and evidence-based practices;

\section{Dovepress}

Interdisciplinary decision-making; Philosophical and ethical issues; Hazard management; Research and opinion for health leadership; Leadership assessment. The manuscript management system is completely online and includes a very quick and fair peer-review system. Visit http://www.dovepress.com/ testimonials.php to read real quotes from published authors.

Submit your manuscript here: http://www.dovepress.com/journal-of-healthcare-leadership-journal 